\title{
Qualitative and Quantitative Evaluation of Image Denoising Techniques
}

\author{
Charandeep Singh Bedi \\ Department of Computer Science \& \\ Engineering \\ Bhai Maha Singh College of \\ Engineering \\ Muktsar, Punjab, India
}

\author{
Dr. Himani Goyal \\ Dean PG Courses \\ Bhai Maha Singh College of \\ Engineering \\ Muktsar, Punjab, India
}

\begin{abstract}
Digital Imaging plays important role in major areas of life such as clinical diagnosis etc. But it faces problem of speckle noise. Speckle noise is referred as 'texture' in medical literature and it may contain useful diagnostic information. Speckle has a negative impact on ultrasound images, as the texture does not reflect the local echogenicity of the underlying scatterers. Physicians generally prefer original noisy images, more willingly than the smoothed versions, even if they are more sophisticated, can destroy some relevant image details. Thus, it is essential to develop noise filters, which can preserve the features that are of interest to the physician. One of the most prevalent cases is distortion due to additive white Gaussian noise, which can be caused by poor image acquisition or by transferring of the image data in noisy communication channels. Moreover there is a long list of image denoising techniques. But problem is that which technique is to be used and for what kind of format. In this paper, we have discussed various spatial filters in chapter 1 . The comparison of the results gives the conclusion and the future scope of the discussion.
\end{abstract}

\section{General Terms}

Digital Image Processing.

\section{Keywords}

Digital Image Processing, Denoising, Speckle noise, Wavelet transform, Spatial filters.

\section{INTRODUCTION}

Digital images play an important role in today's life. It is used in the applications, such as, satellite television, magnetic resonance imaging, ultrasound imaging, geographical information systems, and astronomy and computer tomography. However, one major issue when using this imaging modality is the inherent presence of speckle noise. Its occurrence is often undesirable, since it affects the tasks of interpretation. Ultrasound images suffer from speckle noise, creating images that appear inferior to those generated by other medical imaging modalities. Within each resolution cell, a number of elementary scatterers reflect the incident wave towards the sensor. The back scattered coherent waves which have different phases undergoes constructive or destructive interference in a random manner. The acquired image is thus corrupted by a random granular pattern, called 'speckle', which delays the interpretation of the image.
Speckle filtering is thus a critical pre-processing step in digital imaging providing physicians with enhanced diagnostic ability. Efficient speckle noise removal algorithms may also find applications in real time surgical guidance assemblies. However, it is vital that regions of interests are not compromised during speckle removal [30]. In a recent work [1], we have studied that a successful ultrasound imaging algorithm can achieve both noise reduction and feature preservation if it takes into consideration the true statistics of the signal and noise components. Various filters based upon spatial filtering are observed like Wiener filter, Lee filter, Kuan filter and Median filter. But the discussion is focused on the best image outcome after denoising is done. The approach presented here is totally based upon the comparison of the above said spatial filters for speckle reduction based on the CoC, PSNR and S/MSE parameters.

\section{NOISE REDUCTION IN ULTRASOUND IMAGES}

Some of the best known standard de-speckling filters are the methods of Lee [10], Frost [26] and Kuan . These filters use the second-order sample statistics within a minimum mean squared error estimation approach. Another common de-speckling approach is the homomorphic Wiener filter where the image is first subjected to a logarithmic transform and then filtered with an adaptive filter for additive Gaussian noise [2]. Except these, Median filter is the common a common step in image processing. Its edge-preserving nature makes it useful in cases where edge blurring is undesirable. Since the median value must actually be the value of one of the pixels in the neighbourhood, the median filter does not create new unrealistic pixel values when the filter spans an edge. For this reason the median filter is much better in preserving sharp edges. Median filtering is a non-linear technique that works best with impulse noise (salt \& pepper noise) and speckle noise while retaining sharp edges in the image. The main disadvantage of this technique is that to find the median it is necessary to sort all the values in the neighbourhood into numerical order and this is relatively slow because an extra computation time is needed to sort the intensity value of each set.

Secondly, Lee filter is based on the approach that if the variance over an area is low, then the smoothing will be performed. Otherwise, if the variance is high (e.g. near edges), smoothing will not be performed. Kuan filter is considered to be more superior to the Lee filter. It does not make approximation on the noise variance within the filter window. 
The filter simply models the multiplicative model of speckle into an additive linear form, but it relies on the Equivalent Numbers of Looks (ENL) from an image to determine a different weighted $\mathrm{W}$ to perform the filtering.

$$
\mathrm{W}=(1-\mathrm{Cu} / \mathrm{Ci})(1+\mathrm{Cu})
$$

Where $\mathrm{Cu}$ is the noise variation coefficient and $\mathrm{Ci}$ is the image variation coefficient. Next, the Wiener is a low pass filter that filters an intensity image that has been degraded by constant power additive noise. It uses a pixel wise adaptive wiener method based on statistics estimated from a local neighbourhood of each pixel.

\section{ULTRASOUND IMAGE DENOISING USING SPATIAL FILTERS}

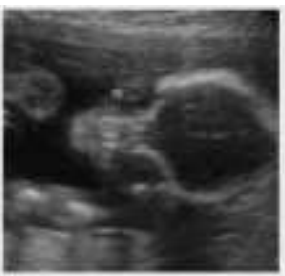

a.

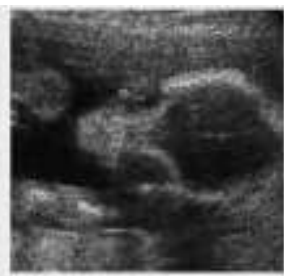

b.

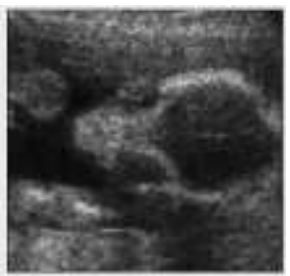

c.
Figure 1. (a) Original Image (b) Noisy Image (c) Filtered Image using Median filter.

Median filter sorts the surrounding pixels value in the window to an orderly set and has replaced the centred pixel within the defined window with the middle value in the set.

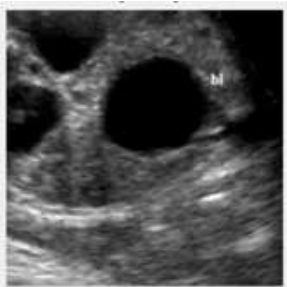

a.

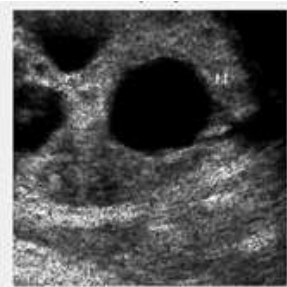

b.

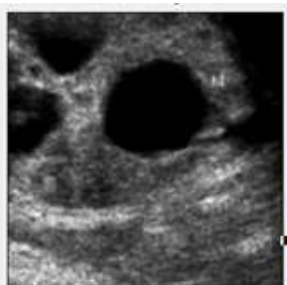

c.
Figure 2. (a) Original Image (b) Noisy Image (c) Filtered Image using Lee filter.

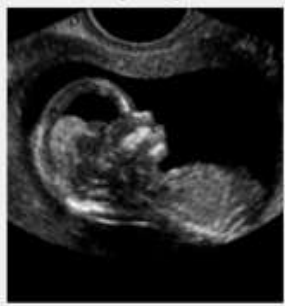

a.

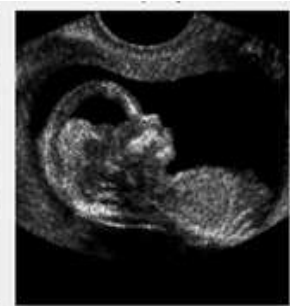

b.

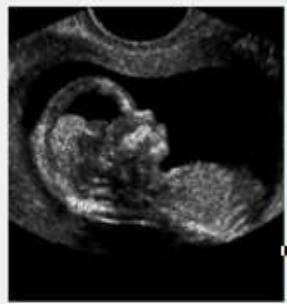

c.
Figure 3. (a) Original Image (b) Noisy Image (c) Filtered Image using Wiener filter.
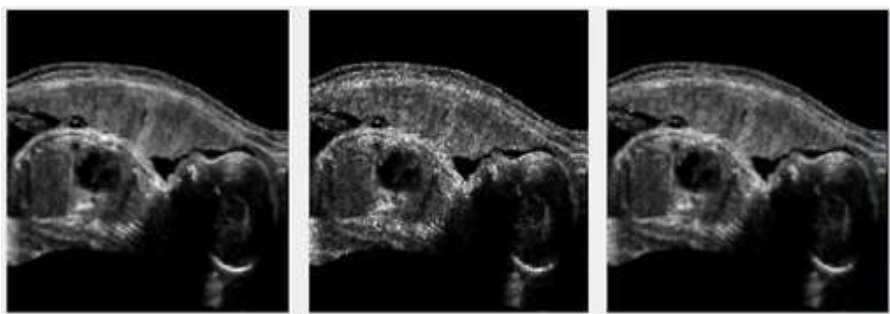

Figure 4. Original Image (a), Noisy Image (b), Filtered Image using Kuan filter.

\section{ULTRASOUND IMAGE DENOISING USING WAVELET TRANSFORM}

Wavelet transform is a tool for improving medical images from noisy data. It consists of a set of basis functions that are used to analyse signals both in time and frequency domains simultaneously. Wavelet denoising attempts to remove the noise present in the signal while preserving the signal characteristics, regardless of its frequency content.

According to wavelet analysis, one of the most effective ways to remove speckle without smearing out the sharp edge features of an ideal image is to threshold only high frequency components while preserving most of the sharp features in the image. The approach is to shrink the detailed coefficients (high frequency components) whose amplitudes are smaller than a certain statistical threshold value to zero while retaining the smoother detailed coefficients to reconstruct the ideal image without much loss in its detail. This process is sometimes called wavelet shrinkage. The schemes to shrink the wavelet coefficients are "keep-or-kill" hard thresholding, and "shrink-or-kill" soft thresholding.
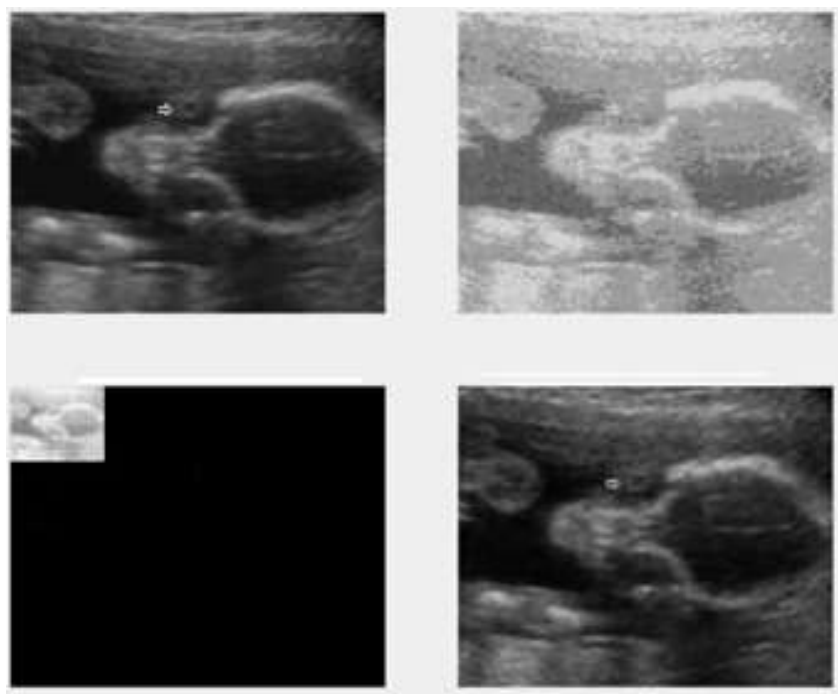

Figure 5. (a) Original Image (b) Noisy Image (c) decomposition image (d) denoised image using Normal Shrink.

Normal Shrink (Figure 5) is an adaptive threshold estimation method for image de noising in the wavelet domain based on the generalized Gaussian distribution (GGD) modelling of sub band coefficients. 
Bayes Shrink is an adaptive data-driven threshold for image denoising via wavelet soft-thresholding. Threshold is driven in a Bayesian framework, and is assumed Generalized Gaussian Distribution (GGD) for the wavelet coefficients in each detail subband.
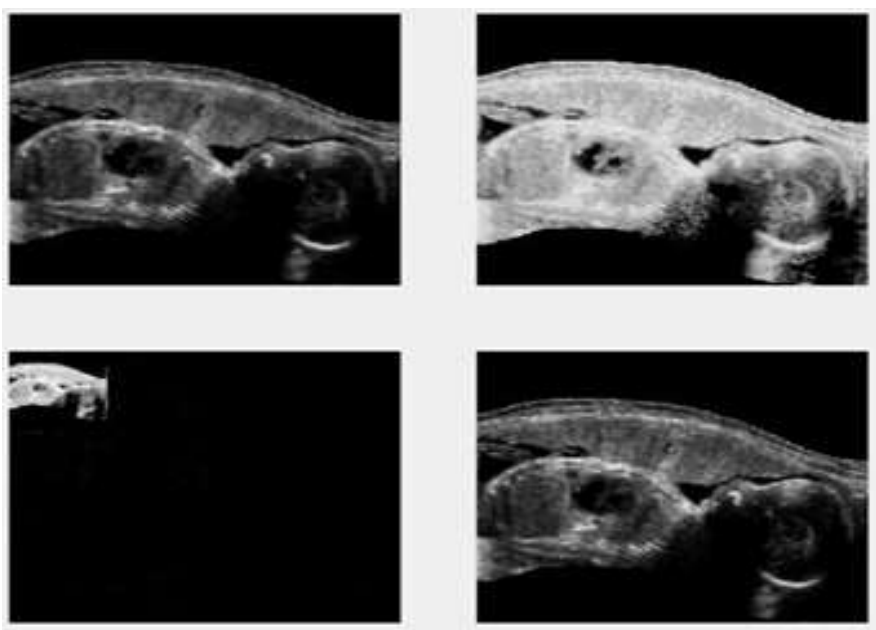

Figure 6. (a) Original Image (b) Noisy Image (c) decomposition image (d) denoised image using Bayes Shrink.

\section{RESULTS}

The four filters have been discussed in the paper. The various parameters collected include the CoC, PSNR and S/MSE which will give the quality related outcomes of the experiment. Peak Signal-to-Noise Ratio (PSNR) is considered to be the least complex metric, as it defines the image quality degradation as a plain pixel by pixel error power estimate. PSNR is an engineering term for the ratio between the maximum possible power of a signal and the power of corrupting noise that affects the fidelity of its representation. In order to quantify the achieved performance improvement, this measure was computed based on the original and the denoised data. Signal to Mean Squared Error for both noisy and de-noised images was identified. The correlation is defined only if both of the standard deviations are finite and both of them are nonzero. It is a corollary of the Cauchy-Schwarz inequality that the correlation cannot exceed 1 in absolute value. The correlation is 1 in the case of an increasing linear relationship and - 1 in the case of a decreasing linear relationship. Its value lies in between in all other cases, indicating the degree of linear dependence between the images. The closer the coefficient is to either -1 or 1 , the stronger the correlation between the images.

Table 1. Comparison of Filters for Ultrasound Images (Jpeg Format)

\begin{tabular}{|l|l|l|l|l|}
\hline $\begin{array}{l}\text { Image } \\
\text { s }\end{array}$ & Filters & PSNR & S/MSE & CoC \\
\hline A. jpg & Median Filter & 30.2064 & 6.1251 & 0.9064 \\
\hline & Lee Filter & 32.2341 & 8.1528 & 0.9467 \\
\hline & Kuan Filter & 29.4931 & 5.4118 & 0.8736 \\
\hline & Wiener Filter & 31.2508 & 7.1695 & 0.9184 \\
\hline & $\begin{array}{l}\text { NormalShrin } \\
\text { k }\end{array}$ & 35.1114 & 11.0301 & 0.9824 \\
\hline & BayesShrink & $\mathbf{3 5 . 2 7 2 3}$ & $\mathbf{1 1 . 1 9 1}$ & $\mathbf{0 . 9 8 3 8}$ \\
\hline
\end{tabular}

Table 2. Comparison of Filters for Ultrasound Images (Tif Format)

\begin{tabular}{|l|l|l|l|l|}
\hline $\begin{array}{c}\text { Image } \\
\text { s }\end{array}$ & \multicolumn{1}{|c|}{ Filters } & \multicolumn{1}{|c|}{ PSNR } & S/MSE & CoC \\
\hline D.tif & Median Filter & 33.1583 & 5.8337 & 0.9798 \\
\hline & Lee Filter & 35.3022 & 7.9775 & 0.9879 \\
\hline & Kuan Filter & 33.9787 & 6.6541 & 0.9854 \\
\hline & Wiener Filter & 33.8325 & 6.5079 & 0.9974 \\
\hline & $\begin{array}{l}\text { NormalShrin } \\
\text { k }\end{array}$ & 33.0141 & 7.85 & 0.973 \\
\hline & BayesShrink & $\mathbf{3 4 . 8 8 3 8}$ & $\mathbf{7 . 9 1}$ & $\mathbf{0 . 9 7 8}$ \\
\hline
\end{tabular}

\section{CONCLUSION}

It have been concluded that amongst all type of spatial filters and wavelet based homomorphic techniques, wavelet based techniques gives better results as compared to spatial filtering techniques. Wavelet based uses a logarithmic transform to separate the noise from the original image. They adopt regularized soft thresholding (wavelet shrinkage) to remove noise energy within the finer scales and nonlinear processing of feature energy for contrast enhancement.

In case of wavelet based denoising methods, noise is removed while preserving the edges with less loss of detail. The main idea is the use of realistic distributions of the wavelet coefficients. By combining these distributions with a simple shrinkage function (soft-thresholding), a closed-form expression for soft thresholding is derived analytically. All the parameters for estimating the threshold are derived automatically from a given ultrasound image.

In future it would be interesting to explore the work in different types of medical images like CT, MRI, and X-ray images collected from hospitals/radiologists may be considered for qualitative and quantitative evaluation. Validation of the work may be done from experts in medical field. Other multi resolution techniques like curve lets may be used instead of wavelets.

\section{ACKNOWLEDGMENTS}

I am highly grateful to Dr. Col. D.S. Grewal, Director/Principal, Bhai Maha Singh College of Engineering, Muktsar, Punjab, for providing this opportunity to carry out the current work. The constant guidance and encouragement received from Dr. Himani Goyal, Assistant Professor \& Dean PG courses, has been of great help in carrying out the present work and is acknowledged with reverential thanks. I would like to express gratitude to other faculty members of Computer Science and Engineering Department, BMSCE for their intellectual support throughout the course of this work.

\section{REFERENCES}

[1] Achim A., Bezerianos A., Tsakalides, P. (2001), "Waveletbased ultrasound image denoising using an alpha-stable prior probability model", International Conference on Image processing, Vol.2, Issue 7-10, pp.221-224.

[2] Achim, A., and Bezerianos, A. (2001), "Novel Bayesian Multiscale Method for Speckle Removal in Medical Ultrasound 
Images", IEEE Transactions on Medical Imaging, Vol. 20, No. 8, pp.772-783.

[3] Arici, T., Dikbas, S. and Altunbasak, Y. (2009), “A Histogram Modification Framework and Its Application for Image Contrast Enhancement", IEEE Transactions on Image Processing, Vol.18, No. 9, pp. 1921-1935.

[4] Arivazhagan, S., Deivalakshmi, S., Kanan, K., Gajbhiye, B., Muralidhar, C., Lukose, S. and Subramanian, M. (2007), "Performance Analysis of Wavelet Filters for Image Denoising", Advances in Computational Sciences and Technology, ISSN 0973-6107, Vol. 1, No. 1, pp. 1-10.

[5] Chang, S., Yu, B., and Vetterli, M. (2000), "Adaptive Wavelet Thresholding for Image Denoising and Compression", IEEE Transactions on Image Processing, Vol. 9, No. 9, pp. 15321546.

[6] Chang, C., Du, Y., Wang, J., Guo, S. and Thouin, P.D. (2006), "Survey and comparative analysis of entropy and relative entropy thresholding techniques", IEEE proceedings on Vision, Image and Signal Processing, Vol.153, Issue 6, pp. 837-850.

[7] Chang, S., Yu, B. and Vetterli, M. (2000), "Spatially adaptive wavelet thresholding with context modeling for image denoising", IEEE Transactions on Image Processing, Vol.9, No.9, pp. 1522-1531.

[8] Chen, G.Y., Bui, T.D. and Krzyzak, A. (2004), "Image denoising using neighbouring wavelet coefficients" IEEE International Conference on Acoustics, Speech, and Signal Processing, Vol. 2, pp. 917-920.

[9] Gonzalez, R. and Woods, R. (2006), "Digital Image Processing with MATLAB", Pearson Prentice Hall, New Delhi, pp. 417-425.

[10] Goossens, B., Pizurica, A. and Philips, W. (2009), "Image denoising using mixtures of projected Gaussian scale mixtures", IEEE Transactions on Image Processing, Vol.18, Issue. 8, pp.1689-1702.

[11] Guo, H., Odegard, J.E., Lang, M., Gopinath, R.A., Selesnick, I., and Burrus, C.S. (1994), "Speckle reduction via wavelet shrinkage with application to SAR based ATD/R," Technical Report CML TR94-02, CML, Rice University, Houston.

[12] Guo, Y., Cheng, H., Tian, J. and Zhang, Y. (2009), "A Novel Approach to Speckle Reduction in Ultrasound Imaging”, Ultrasound in Medicine \& Biology, Vol.35, Issue 4, pp. 628-640.

[13] Gupta, S., Chauhan, R. and Sexena, S. (2004), “A WaveletBased Statistical Approach for Speckle Reduction in Medical Ultrasound Images", Medical and Biological Engineering \& Computing, Vol. 42, No.2, pp. 189-192.

[14] Gupta, S., Chauhan, R.C., and Saxena, S.C. (2005), “A robust multi-scale non-homomorphic approach to speckle reduction in medical ultrasound images", IEE J. Int. Fed. Med. Biol. Eng. 152 (1) pp.129-135.

[15] Hamza, A. and Krim, H. (2001), "Image Denoising: A Nonlinear Robust Statistical Approach", IEEE Transactions on Signal Processing, Vol. 49, No. 12, pp. 3045-3054.

[16] Jain, A. (1989), "Fundamentals of digital image processing", Prentice-Hall Inc., Englewood Cliffs, New Jersey.
[17] Kaur, L., Gupta, S. and Chauhan, R. (2002), "Image Denoising Using Wavelet Thresholding", Third Indian Conference on Computer Vision, Graphics and Image Processing, Ahmedabad, pp.1-4.

[18] Lian, N.X., Zagorodnov, V. and Tan, Y.P.(2005), "Color image denoising using wavelets and minimum cut analysis" IEEE Signal Processing Letters, Vol. 12, Issue 11, pp.741-744.

[19] Mastriani, M. (2009), "Denoising and Compression in Wavelet Domain via Projection onto Approximation Coefficients" International Journal of Signal Processing Vol.5, No.1, pp.20-30.

[20] Mastriani, M., and Giraldez, A.E. (2005), "Smoothing of coefficients in wavelet domain for speckle reduction in Synthetic Aperture Radar images" ICGST-GVIP Journal, Vol.5. Issue 6. pp. 1-8.

[21] Mohideen, S., Peruma, S., Sathik, M. (2008), "Image Denoising using Discrete Wavelet transform", International Journal of Computer Science and Network Security, Vol. 8, No.1, pp.213-216.

[22] Nadernejad, E., Karami, M.R., Sharifzadeh, S and Heidari, M. (2009), "Despeckle Filtering in Medical Ultrasound Imaging”, Contemporary Engineering Sciences, Vol. 2, No.1, pp. $17-36$.

[23] Pizurica, A., Wink, A., Vansteenkiste, E., Philips and W. Roerdink, B.T.M (2006), "A Review of Wavelet Denoising in MRI and Ultrasound Brain Imaging," Current Medical Imaging Reviews, Vol. 2, No. 2, pp. 247-260.

[24] Pizurica, A., Philips, W., Lemahieu, I., and Acheroy, M. (2003), "A Versatile Wavelet Domain Noise Filtration Technique for Medical Imaging," IEEE, Transactions on Medical Imaging, vol. 22, No. 3, pp. 323-331.

[25] Saad, A. (1996), "Speckle filtering in SAR images by contrast modification, comparison with a large class of filters", Annals of telecommunications, Vol.51, No. 5, pp. 233-244.

[26] Sudha, S., Suresh, G. and Sukanesh, R. (2009), "Speckle noise reduction in ultrasound images using context-based adaptive wavelet thresholding", IETE journal, Vol. 55, pp 135143.

[27] Watson, A.B., Yang, G.Y., Joshua A. Solomon, J.A. and Villasenor, J. (1997), "Visibility of Wavelet Quantization Noise" IEEE Transactions on Image Processing, Vol. 6, No. 8, pp.1164 1175.

[28] Xuli Z., Laine, A.F., Geiser, E.A. (1998), "Speckle reduction and contrast enhancement of echocardiograms via multiscale nonlinear processing", IEEE Transactions on Medical Imaging, Vol. 17 No.4, pp. 532-540.

[29] Zhong, S. and Cherkassky, V. (2000), "Image denoising using wavelet thresholding and model selection", International conference on Image Processing, Vol.3, pp. 262-265.

[30] Sudha, S., Suresh, G.R., and Sukanesh, R. (2009), "Speckle Noise Reduction in Ultrasound Images by Wavelet Thresholding based on Weighted Variance" International Journal of Computer Theory and Engineering, Vol. 1, No. 1, pp.1793-8201. 